\title{
Coexistence of lupus nephritis and sickle cell trait, an electron microscopic assessment of renal glomerular damage: Case report of a rare association
}

\author{
Yahya Elficki ${ }^{1}$, Atif Rawas², Asseil Ali Bossei ${ }^{3}$, Areej Bdawod ${ }^{3}$, Reem Zabani ${ }^{3}$, Bayan Shams ${ }^{3}$
}

${ }^{1}$ Consultant of Internal Medicine, FRCP-UK, King Abdulaziz Hospital, Jeddah, Kingdom of Saudi Arabia

${ }^{2}$ Research Assistant and Coordinator, MD, King Abdulaziz Hospital, Jeddah, Kingdom of Saudi Arabia

${ }^{3}$ Research Assistant and Coordinator, MBBS student, Ibn Sina National College for Medical Science, Jeddah, Kingdom of Saudi Arabia

\section{Type of article: Case report}

\begin{abstract}
Because of similarities of the musculoskeletal, central nervous system, and renal manifestations in both diseases, diagnosing systemic lupus erythematosus (SLE) in sickle cell disease (SCD) patients can be difficult to establish. Although Sickle Cell Trait (SCT) is still considered a benign form of SCD, its impact on kidney injury and other renal manifestations is well recognized in the literature. In this case report, we look at the challenges that develop when diagnosing patients with a concurrence of both diseases and the importance of early recognition and treatment of lupus nephritis in SCT patients. We present a case of a male patient with sickle-cell trait who was admitted to our hospital complaining of low grade fever and pancytopenia for investigations proven to be SLE. A renal biopsy on electron microscopy assessment with different staining modalities as well as immune fluorescence revealed mixed pathological changes. We emphasize the importance in considering the presence of a coexisting autoimmune disease in a patient with sickle hemoglobinopathies even in the milder forms like SCT which may display an atypical and/or multisystem presentation. Also, the impact of the two conditions on the renal pathological changes should be expected to be more damaging even at early onset of SLE flare, and hence, an urge to start with more intensified immunosuppressive medications.
\end{abstract}

Keywords: Systemic lupus erythematosus, Lupus nephritis, Sickle cell trait, Aplastic anemia, Febrile neutropenia

\section{Introduction}

SLE (systemic lupus erythematosus) is the commonest multisystem connective tissue disease. It is a chronic inflammatory disease of unidentified etiology which can affect the skin, joints, lungs, kidneys, nervous system, the serous membranes as well as other organs of the body. Presence of SLE is worldwide but its prevalence varies, with most prevalence $(1: 250)$ observed in African American women. Its prevalence varies in other populations to $1: 10000$. SLE is rare in males, as $90 \%$ of cases are in women, usually at child bearing age; but it can also be sometimes found in children, men, and the elderly (1). Renal involvement can be detected in approximately $50 \%$ of patients following renal biopsy. Renal involvement usually occurs in the first few years of illness. Different forms of glomerulonephritis may develop, and renal biopsy is useful in defining the type and extent of renal involvement (2). However, sickle cell trait (SCT) is a major cause of mortality in patients with sickle cell disease (SCD) (3). Renal manifestations of SCT include: urinary tract infection, hematuria, hyposthenuria, renal medullary carcinoma, renal papillary necrosis, and/or renal infarcts at high altitude. Only few case reports in the literature had been focusing on the association between SCD and SLE and the early impact of the two diseases on the kidney, since SCT is known to have multiple verities of renal damage (despite being the mildest and most asymptomatic form of SCD subtypes); Large prospective epidemiological studies are necessary to determine the prevalence and rate of progression of renal damage when overlap of immune complex diseases occurs in patients with SCD especially the SCT variant (4).

\section{Corresponding author:}

Asseil Ali Bossei, Ibn Sina National College for Medical science, Jeddah, Kingdom of Saudi Arabia.

Tel.: +966551500666, E-mail: Asseil.a.b.95@gmail.com

Received: June 09, 2017, Accepted: July 11, 2017, Published: September 2017

iThenticate screening: July 12, 2017, English editing: August 27, 2017, Quality control: September 10, 2017

(C) 2017 The Authors. This is an open access article under the terms of the Creative Commons Attribution-NonCommercialNoDerivs License, which permits use and distribution in any medium, provided the original work is properly cited, the use is non-commercial and no modifications or adaptations are made. 


\section{Case presentation}

\subsection{Clinical presentation}

A 27 year old male had been admitted to our hospital twice within one month. At first admission, presentation was a low grade fever for three weeks and pancytopenia for investigations. There was no previous medical history of any illnesses apart from family history of sickle cell anemia. On examination, the patient was conscious oriented, alert, had a pulse rate of $90 / \mathrm{min}$, regular, temperature $38^{\circ} \mathrm{C}$, and blood pressure was $118 / 70 \mathrm{mmHg}$. He looked pale and mildly jaundiced, no lymph nodes enlargement, rash, bleeding, nor lower limbs edema. Normal first and second heart sounds, regular and equal peripheral pulsations. Abdomen was soft, lax, no tenderness, no organomegaly, Chest was normal vesicular breathing with average air entry bilaterally, and neurological examination was unremarkable.

\subsection{Laboratory}

A few days later, the patient started to develop puffy eyes and lower limbs edema with deterioration of his creatinine level, nephrotic syndrome was suspected and further investigation showed serum creatinine, 109 (2 days later 187 umol/l), serum Proteins $70 \mathrm{gm} / \mathrm{dL}$; serum albumin $26 \mathrm{gm} \%$, 24 hours urinary proteins was $1.4 \mathrm{gm}$, serum Bilirubin was $47 \mathrm{mg} / \mathrm{dL}$, serum Alanine Amino Transferase was 30 units/L, random blood Sugar was $131 \mathrm{mg} / \mathrm{dL}$, serum sodium $126 \mathrm{meq} / \mathrm{L}$ and serum potassium $3.9 \mathrm{meq} / \mathrm{L}$ and positive sickling test was noted at 24 hours. His hemoglobin was $8 \mathrm{gm} / \mathrm{dL}$ reached down to 6.9 (in 2 days); total leukocyte count was 1.8 (reached 0.79 in 2 days) with differential count showing neutrophils 0.41 , lymphocytes 30 and eosinophils and monocytes $0 \mathrm{k} / \mathrm{uL}$, platelets $75,000 / \mu$. reticulocyte count was below $1 \%$. Hb electrophoresis ( $\mathrm{HbS} \mathrm{35 \% ,} \mathrm{HbF} \mathrm{0.2,} \mathrm{HbA} 60 \%, \mathrm{HbA} 23.2 \%$ ) Peripheral smear showed (Microcytic hypochromic with few target cells, and elliptocytes with reuleaux formation, thrombocytopenia with severe leucopenia, neutropenia and lymphopenia (Reticulocyte count raised up to 17\%), Direct Coombs test +ve, routine serology for HBV, HCV, HIV, CMV and monospot for EBV were all negative. Autoimmune screening showed positive ANA titer 1/ 620 and anti-double stranded DNA titer 1/ 1250 with low C3 and C4, CRP 35, ESR $85 \mathrm{~mm}$, sickle cell trait and systemic lupus erythematosus diagnosis was settled and the patient started bolus methylprednisolone and hydroxychloroquine with subsequent rise in his leucocytes and platelets count as well as full recovery of the renal function tests.

\subsection{Follow up}

The patient was discharged upon his request to follow up at nephrology, rheumatology, and general medicine outpatient clinics. One month later, upon recurrence of fever, and recurrence of generalized edema, the patient was readmitted and investigations at second admission revealed: His hemoglobin had dropped again down to $8 \mathrm{gm} / \mathrm{dL}$, total leukocyte count was 0.95 with differential count showing neutrophils 0.8 then dropped down to 0.5 . Reticulocyte count was below 1\%. Platelet 10,000, second peripheral blood smear revealed: red cells were normocytic normochromic, rouleaux formation with target cells and elliptocytes. There was severe leucopenia, neutropenia and lymphopenia and thrombocytopenia. Serum creatinine $115 \mathrm{umol} / \mathrm{l}$, serum proteins $66 \mathrm{gm} / \mathrm{dL}$; serum albumin $18 \mathrm{gm} \%$, serum bilirubin was $47 \mathrm{mg} / \mathrm{dL}$, serum alanine amino transferase 25 units/L, serum sodium 133 $\mathrm{meq} / \mathrm{L}$ and serum potassium $4.4 \mathrm{meq} / \mathrm{L}$ urinary 24 hours for protein was $2.2 \mathrm{gm}$. The patient was started on: prednisolone, frusemide, perindopril, atorvastatin, ceftriaxone, moxifloxacillin, repeated blood transfusions and rehydration as well as thromboprophylaxis. The patient developed photosensitivity, and hydroxychloroquine induced rash. Among investigations in the second admission, abdominal ultrasonography showed mild hepatosplenomegaly but both kidneys were of average size, parenchymal echo-pattern, cortical thickness no stones nor backpressure could be noted. Upon diagnosis of febrile neutropenia, pancytopenia, and nephrotic syndrome the patient was admitted at reversed isolation room and antibiotics were modified to treatment guidelines for severe neutropenia as well as other conservative management and close observation, together with more frequent serials of blood works. Bone marrow biopsy revealed H \& E section showed hypercellular bone marrow. Megakaryopoiesis was adequate as many megakaryocytes were seen. Most specimens showed crushed cells. From other small crushed areas, erythroid precursor was detected, but reduced and myeloid cells were markedly decreased. After recovery from neutropenia and pancytopenia, renal biopsy was done as the patient had significant proteinuria. Kidney biopsy Gross Description: It consisted of two thin cores of grayish-tan soft tissue measuring 1.2 and $1.4 \mathrm{~cm}$ in length. Both cores were less than $0.1 \mathrm{~cm}$ in width. Microscopic description of the tissue examined with H\&E, PAs Trichrome, and Jones silver stains, The biopsy consisted of renal cortex and medulla. Twenty-seven glomeruli were present for evaluation none revealed global or segmental sclerosis. The glomeruli showed moderate mesangial matrix expansion and hypercellularity associated with focal thickening of the capillary wall. No endocapillary proliferation was seen. There was mild interstitial fibrous and tubular atrophy (5-10\% of the cortical tissue) associated with mild focal interstitial mononuclear inflammatory cell infiltrate composed of lymphocytes and plasma cells. Arteries and arterioles were unremarkable. Medullary tissue was unremarkable as well. Immunofluorescence revealed three 
glomeruli were present for evaluation. The glomeruli revealed granular mesangial staining with antisera specific for $\operatorname{IgG}(1+), \operatorname{IgA}(1+), \operatorname{IgM}$ (trace), C3 (1+), C1q (2+). No staining was seen with fibrinogen. Electron microscope study showed the semi thin sections revealed two glomeruli. Ultra-structural examination demonstrated glomerular basement membranes to be mostly normal except for focal duplication with mesangial interposition. There were scattered subendothelial, subepithelial, intramembranous and mesangial immune complex type electron dense deposits. Rare large hump-like subepithelial deposits were also seen. The podocytes showed focal foot process effacement. Diagnosis: Focal lupus nephritis, ISN/RPS class III, global glomerulosclerosis (0/27), segmental sclerosis $(0 / 27)$, interstitial fibrosis and tubular atrophy. The glomerular changes in this biopsy were rather mild on light microscopic examination and resembled changes of class 11 lupus nephritis. However, electron microscopic examination revealed significant numbers of electron dense deposits along the peripheral capillary loop with focal duplication of the basement membranes. In view of these changes the glomerular findings were most compatible with class III lupus nephritis. Upon adding mycophenolate mofetil (MMF), IV gamma globulins, and continuation of high doses of prednisolone, plasmapheresis; the patient's general condition dramatically improved over a fifteenday period with total recovery of his blood count readings, as well as the proteinuria, edema, and kidney function tests; A scheduled follow-up plan at outpatient's nephrology and rheumatology clinics was arranged before discharge.

\section{Discussion}

As described in this case, the recognition of SLE is often difficult and delayed when there is a coexistence of both diseases. The diagnosis of SCT is usually made several years prior to the onset of SLE. Both SCT and SLE are chronic, progressive diseases with diverse renal manifestations that show a significant overlap in presentation. This accounts for the diagnostic difficulty that may arise with the concurrence of SLE and SCT (5). Furthermore, approximately $20 \%$ of SCD patients have positive ANA antibodies with titers greater than 1/160, making the diagnosis more challenging in clinical practice (6). However, no studies had been found in the literature focusing on prevalence of autoantibodies and immune complexes diseases in the SCT variant type of SCD. Occurrence of connective tissue diseases such as systemic lupus Erythematosus (SLE) in adult patients with SCD appears to be increasing, according to recent observational cohort studies. The precise reasons for the increased risk are still unknown, but patients with sickle cell disease face the risk of infections due to dysfunction of the immune system. Furthermore, association with regulatory B cells (Breg) is possible, as these cells suppress any inflammatory responses, sustain tolerance and prevent the progress of SLE in animal models through the production of interleukin10 (IL-10) (7). Generally, patients who have SCA are known to develop idiopathic nephrotic syndrome with focal and segmental glomerulosclerosis or with membranoproliferative glomerulonephritis. Incidence of SLE is documented in adults and is being more frequently reported in children with SDE $(5,8)$. But, lupus nephritis with sickle cell nephropathy is rarely documented in adults, and it is suggested that a defect in the alternative pathway of complement activity seen in patients with SCD may predispose immune complex disorders (9). A broad spectrum of systemic complications can be associated with sickle cell disease and/or systemic lupus erythematosus (10, 11). Unless serological work-up is carried out, mesangial expansion and basement membrane duplication, as well as occurrence of immune complex disorders in sickle cell disease may delay the exposure of lesions due to systemic lupus erythematosus (3). Despite being described as a mild, dormant, and/or benign form of SCD, sickle cell trait, is an inherited condition marked by having a single copy of the sickle cell gene but not the two copies needed to cause sickle cell disease (SCD). Yet, SCT may raise the risk of chronic kidney disease (1). Although it is understood that SCT is linked with hematuria and renal papillary necrosis, the precise connection between SCT and CKD has yet to be determined (2). Possible pathophysiological mechanisms for kidney damaged patients with sickle cell disease have been defined (3). Chronic reversible sickling induced by hypoxia in the renal medulla, results in ischemia and micro infarction of the renal tubules. Local ischemia and hemolysis cause release of vasoactive factors and promote glomerular hyperfiltration, ultimately resulting in glomerulosclerosis and proteinuria. Researchers collected data on 15,975 self-identified African-American participants, 1,248 with SCT and the rest were non-carriers of the sickle gene. They looked specifically at how many members of each group had developed chronic kidney disease or other markers of impaired kidney function, including poor ability to filter blood through the kidneys (hyposthenuria) and the presence of protein in urine. The incidence of chronic kidney disease was significantly higher in those with SCT than in non-carriers: about $20.7 \%$ in those with the trait, versus $13.7 \%$ of those without it (4). In the SCT carriers, the researchers hypothesize that although blood cells are less likely to sickle - or warp into a sickle shape - as severely or frequently in individuals with SCT as they do in patients with SCD, they can still experience infrequent and localized sickling that might affect certain organs more than others, clogging blood vessels and restricting oxygen to these areas. This mild sickling may be enough to eventually damage the kidneys, they say, leading to chronic kidney disease and other kidney problems (12). Furthermore, systemic lupus erythematosus is a complex 
autoimmune disorder which has heterogeneous disease course and clinical manifestations. Its distinctive features are its dysregulated adaptive immune pathways and progression of anti-nuclear antibodies (13). Lupus nephritis is a severe manifestation of SLE which has a complex pathogenesis. Generating lupus nephritis requires more than immune complex formation and complement activation alone (7). Disruption of the gamma chain of the $\mathrm{Fc}$ receptor in New Zealand Black and New Zealand White impeded severe glomerulonephritis while immune complex deposition continued to occur (7). The genetic vulnerability factor to SLE of Fc gamma receptor type IIA R131 in humans, may cause failure of clearance of an IgG2 anti-C1q antibody to the collagen-like region of C1q complex, leading to glomerulonephritis (7). In tests carried out on Egyptian SLE patients, association of an Fc gamma type IIB homozygous genotype (Thr/Thr) with increased risk of SLE and complications of nephritis was observed, however, the Fc receptor type IIA polymorphisms were not associated in Egyptian SLE patients (7). Recombinant human soluble Fc gamma receptors (CD32) bound IgG coated murine erythrocytes, decreasing phagocytosis by macrophages via disruption of the IgG and $\mathrm{Fc}$ gamma receptor interaction (7). Although CD32 treatment failed to reverse nephritis, onset of proteinuria was delayed and survival time increased (7). It was found that a synthetic peptide from the membrane-proximal extracellular domain of Fc gamma receptor II is a competitive inhibitor of IgG binding recombinant $\mathrm{Fc}$ receptor II in vitro and it increased survival time while decreasing proteinuria in MRL/lpr mice in vivo (7). When taken together, it appears that glomerular deposition of C1q in the context of immune complexes, complement activation, and functional $\mathrm{Fc}$ gamma receptors are required to cause renal damage (7). This review studied the various functions of complement, autoantibody and apoptosis, and the adaptive immune system in contributing to the pathogenesis of lupus nephritis. Complement aids in opsonizing immune complexes for degradation by effector immune cells, and deficiency in early components of the complement cascade have association with active disease (7). Molecules normally contained within cells can become accessible to autoantibody with inefficient apoptotic clearing and the breaking of tolerance can occur. By activating auto-reactive $\mathrm{B}$ cells and instigating a $\mathrm{T}$ cell response, the innate immune system may induce the adaptive immune system through toll-like receptors. Products of the adaptive immune response might serve as predictors for disease activity and through blocking activation of specific components of the adaptive immune, there may be therapeutic effects in controlling inflammation (7). The current treatment approach includes steroidal and non-steroidal anti-inflammatory agents and immunosuppressive drugs, antimalarial medication, as well as azathioprine, cyclophosphamide, methotrexate and mycophenolic (MMF) acid (13). Although there has been considerably advanced improvement in prognosis for SLE patients, overcoming the difficulties in treating patients with active disease refractory to traditional therapies continues to be a challenge. Not too far into the future are, new targeted therapies specially designed to block pathways involved in disease pathogenesis (13). As knowledge of the initiation and progression of the disease grows, we can look forward to therapeutic possibilities that focus on blocking defined phases of disease pathogenesis (13). A large number of patients do not attain remission using regular procedures combining cyclophosphamide or MMF. Furthermore, side effects are quite common and patients may flare despite going into remission. Consequently, a number of alternative regimens have been explored for use, either as supplementary therapy or as substitutions. Regrettably, most of these studies are either observational or uncontrolled (14). As patient survival has improved with increasing efficacy of immunosuppressive and supportive therapy, cardiovascular events have become more important cause of mortality in patients with lupus rather than the previous end stage renal disease known causes of mortality (14). Ongoing tubulointerstitial inflammation and fibrosis which cause additional renal impairment (14) are a result of persistent proteinuria. Optimal control of BP, hyperlipidemia, and proteinuria, along with cessation of cigarette smoking, may be beneficial in managing lupus nephritis. It has been shown that using angiotensin converting enzyme inhibitors and angiotensin receptor blockers will reduce the proteinuria and decelerate the development of glomerular diseases, including lupus nephritis (14). In patients with lupus nephritis, the target BP should be $<130 / 80$.

It is recommended that aggressive control of lipids with statins be undertaken. Statins, as well as lowering lipid levels, may have positive effects on endothelial cells and B cells, which will lead to reduced levels of anti-dsDNA antibodies and proteinuria, possibly slowing the progression of underlying renal disease. Antimalarial drugs like hydroxychloroquine (HCQ) are shown to impede lupus flares, prolong survival, and guard against irreversible organ damage (14). In a study of the LUMINA cohort of SLE patients, those who received HCQ (79.3\%) displayed a lower incidence of WHO class IV GN, had lower disease activity, and were given lower glucocorticoids doses than those who did not receive HCQ (14). In SLE patients, use of alternative and complementary medical therapy is common (up to $50 \%$ in the United States); however, only a small number of randomized trials support this practice (14). In conclusion, lupus nephritis that does not respond to initial therapy is linked with poorer long-term prognosis. While the evidence is not established upon optimal scientific studies, a number of therapeutic approaches may be tried with varying degrees of accomplishment, including extending the course of standard therapy, converting to 
MMF or intravenous cyclophosphamide, using adjunctive plasmapheresis or immunoadsorption, including a CNI, and using B cell-targeted therapy with rituximab (14). Strict vigilance and preventive measures against infection are mandated when immunosuppressant is increased (15).

\section{Conclusions}

Systemic lupus erythematosus and sickle cell disease are chronic diseases that have several clinical and laboratory findings in common, which leads to difficult diagnosis and difficulty in finding the correct treatment. Although the association between these diseases is not common, SCA with SLE is known in children and less common in adults, diffuse proliferative lupus nephritis with sickle cell nephropathy has rarely been reported in adults, we highlight that physicians who treat such diseases should be alert to this possibility even with the milder variants such as sickle cell trait, since it requires early diagnosis and more aggressive management.

\section{Acknowledgments:}

We thank our colleagues from Ibn Sina National College for Medical Studies who provided insight and expertise that greatly assisted the research revision for publication. Also, we would like to thank our colleagues from King Abdul Aziz Hospital who provided insight and expertise that greatly assisted the research.

\section{Conflict of Interest:}

There is no conflict of interest to be declared.

\section{Authors' contributions:}

All authors contributed to this project and article equally. All authors read and approved the final manuscript.

\section{References:}

1) Ismail Hossain M, Sarwer jahan SM, Ashraful haque M, Mobasher Alam ABM, Ahmed M, Zakir Hossain M. Systemic lupus Erythematosus in male: two case reports. Bangladesh Journal of Medicine. 2013; 24(2).

2) Schur PH. Systemic Lupus Erythematosus (SLE). John Wiley \& Sons; 2015: 1-11. doi: 10.1002/9780470015902.a0002147.pub3.

3) Kanodia KV, Vanikar AV, Goplani KR, Gupta SB, Trivedi HL. Sickle cell nephropathy with diffuse proliferative lupus nephritis. Diagnostic Pathology. 2008; 3: 9. doi: 10.1186/1746-1596-3-9.

4) Emilia Chalayer, Martine F French, Pascal Cathébras

5) Saxena VR, Mina R, Moallem HJ, Rao SP, Miller ST. Systemic lupus erythematosus in children with sickle cell disease. J Pediatr Hematol Oncol. 2003; 25(8): 668-71. doi: 10.1097/00043426-200308000-00019. PMID: 12902927.

6) Stuart MJ, Nagel RL. Sickle cell disease. Lancet. 2004; 364(9442): 1343-60. doi: 10.1016/S01406736(04)17192-4.

7) Flanagan G, Packham DK, Kincaid-Smith P. Sickle cell disease and the kidney. Am J Kidney Dis. 1993; 21(3): 325-7. doi: 10.1016/S0272-6386(12)80755-4.

8) Shariff S, Al-Hemi B, Al Yousif R, Jabreel N, Al Gharreb S, Balaji D. Spectrum of renal disease in sickle cell anemia. Indian J Nephrology. 2006; 16: 157-63.

9) Khalidi NA, Ajmani H, Varga J. Coexisting systemic lupus erythematosus and sickle cell disease: a diagnostic and therapeutic challenge. J Clin Rheumatol. 2005; 11(2): 86-92. doi: 10.1097/01.rhu.0000158549.92844.96.

10) Saborio P, Scheinman JI. Sickle cell nephropathy. J Am Soc Nephrol. 1999; 10(1): 187-92. PMID: 9890326.

11) Naik RP, Lanzkron S, Morgan E, Grams ME, Coresh J. Sickle Cell 'Trait' Linked to Chronic Kidney Disease. JAMA. 2014.

12) Yildirim-Toruner C, Diamond B. Current and novel therapeutics in the treatment of systemic lupus erythematosus. J Allergy Clin Immunol. 2011; 127(2): 303-12. doi: 10.1016/j.jaci.2010.12.1087. PMID: 21281862 , PMCID: PMC3053574.

13) Kalloo S, Aggarwal N, Mohan P, Radhakrishnan J. Lupus Nephritis: Treatment of Resistant Disease. Clin J Am Soc Nephrol. 2013; 8: 154-61. doi: 10.2215/CJN.05870612. PMID: 23296380.

14) Sterner RM, Hartono SP, Grande JP. The Pathogenesis of Lupus Nephritis. J Clin Cell Immunol. 2014; 5(2): 205. doi: 10.4172/2155-9899.1000205. PMID: 25133068, PMCID: PMC4131745.

15) Weening JJ, D'Agati VD, Schwartz MM, Seshan SV, Alpers CE, Appel GB, et al. The Classification of Glomerulonephritis in Systemic Lupus Erythematosus Revisited. J Am Soc Nephrol. 2004; 15: $241-50$. PMID: 14747370. 